\title{
Comparing Efficacy of Analgesic Modalities in Patients Undergoing Total Knee Arthroplasty [Letter]
}

\author{
Ying Gao (D), Fu-Shan Xue (D), Cheng-Wen Li $\mathbb{D}$ \\ Department of Anesthesiology, Beijing Friendship Hospital, Capital Medical University, Beijing, People's Republic of China
}

Correspondence: Fu-Shan Xue, Department of Anesthesiology, Beijing Friendship Hospital, Capital Medical University, 95 Yong-An Road, Xi-Cheng District, Beijing 100050, People's Republic of China, Tel +86-139-I I 17-7655, Fax+86-10-63138362, Email xuefushan@aliyun.com; fushanxue@outlook.com

\section{Dear editor}

In a noninferiority randomized controlled trial with 88 patients undergoing elective total knee arthroplasty (TKA), Xiao et al $^{1}$ showed that dexmedetomidine (Dex) combined with femoral nerve block (FNB) provided similar analgesic efficacy to FNB combined with sciatic nerve block (SNB). Other than the limitations described in the discussion, however, we noted other issues in the design and results of this study that deserve further discussion and clarification.

First, the authors used the results of their preexperiment for sample-size estimation, but the readers were not provided the sample size of this preexperiment. Most importantly, it was unclear whether the results of the preexperiment were included in the final data analysis. Furthermore, this study applied the design of a noninferiority randomized controlled trial. However, the authors did not clearly describe why a 30\% difference in the primary end point was selected as the noninferiority margin to demonstrate equivalence between two interventions. When a noninferiority randomized controlled trial is designed to determine the clinical efficacy of a new treatment, it is generally considered that a slight improvement in patient condition should be deemed clinically important if this new treatment is expected to be safe and convenient. $^{2}$ For patients undergoing total hip and knee arthroplasty, the recommended minimal clinically important differences in pain score in the literature are really 1.5 at rest state and 1.8 during motion on a $0-10$ pain visual analogue scale. ${ }^{3}$ We noted in particular that the net between-group difference of mean pain levels on the visual analogue scale during motion at postoperative 24 hours was only 0.3 in their preexperiment, which is significantly smaller than their expected noninferiority margin of a $30 \%$ difference. Therefore, we argue that a sample of 44 patients in each group seems impossible to achieve an effective inferiority test.

Second, by comparing the proportion of patients without rescue-analgesic requests up to postoperative 48 hours and the dosages of different rescue analgesics (oxycodone, tramadol, diclofenac sodium, and lidocaine), the authors concluded that Dex combined with FNB decreased the doses of postoperative rescue analgesics to the extent comparable to combined SNB with FNB. This seems a little inappropriate. When postoperative analgesic consumption is compared, it is commonly required that equianalgesic conversion of all analgesics used for postoperative pain control should be performed to report opioid consumption by milligram morphine equivalent in oral or intravenous form, as performed in other studies assessing the efficacy of different analgesic modalities for postoperative pain control in patients undergoing TKA. ${ }^{4-6}$ Furthermore, the recommended minimal clinically important difference for postoperative opioid consumption is an absolute reduction of $10 \mathrm{mg}$ intravenous morphine in morphine milligram equivalent of 24-hour opioid consumption. ${ }^{3}$

Finally, the subjects were geriatric patients with a mean age $>65$ years. The two groups were comparable in terms of first time to get out of bed and walk, occurrence of postoperative delirium, incidence of postoperative nausea and vomiting, and length of hospital stay, but the authors did not assess the quality of early postoperative recovery and patients' satisfaction with postoperative analgesia, as performed in other studies. ${ }^{4,6} \mathrm{In}$ fact, these two variables are very important for determining efficacy and clinical availability of an intervention for postoperative pain control. ${ }^{7}$ 
Furthermore, they are easily measured using using the QoR-15 and a 0-10 visual analogue scale where 0 represents least satisfied and 10 most satisfied, respectively. The QoR-15 is a comprehensive score derived from a patient questionnaire in which 15 questions on various clinically relevant domains of postoperative recovery, such as general well-being, mood, pain, sleep quality, and nausea and vomiting, are elaborated. The maximun score on each question is 10 and overall 150 , with higher scores indicating better quality of postoperative recovery. ${ }^{8}$ We believe that this study would have provided more useful data regarding efficacy and clinical values of Dex combined with FNB for pain control after TKA if the design had included measurements on the quality of recovery and patient satisfaction with postoperative analgesia.

\section{Disclosure}

The authors report no conflicts of interest in this communication.

\section{References}

1. Xiao R, Liu LF, Luo YR, et al. Dexmedetomidine combined with femoral nerve block provides effective analgesia similar to femoral nerve combined with sciatic nerve block in patients undergoing total knee arthroplasty: a Randomized Controlled Study. Drug Des Devel Ther. 2022;16:155-164. doi:10.2147/DDDT.S334415

2. Christensen E. Methodology of superiority vs. equivalence trials and non-inferiority trials. $J$ Hepatol. 2007;46(5):947-954. doi:10.1016/j. jhep.2007.02.015

3. Laigaard J, Pedersen C, Rønsbo TN, Mathiesen O, Karlsen APH. Minimal clinically important differences in randomised clinical trials on pain management after total hip and knee arthroplasty: a systematic review. Br J Anaesth. 2021;126(5):1029-1037. doi:10.1016/j.bja.2021.01.021

4. Wang Q, Hu J, Zeng Y, Li D, Yang J, Kang P. Efficacy of two unique combinations of nerve blocks on postoperative pain and functional outcome after total knee arthroplasty: a Prospective, Double-Blind, Randomized Controlled Study. J Arthroplasty. 2021;36(10):3421-3431. doi:10.1016/j. arth.2021.05.014

5. Kjærsgaard-Andersen P, Rasmussen LE, Pleckaitiene L, Foss NB. High-dose steroids in high pain responders undergoing total knee arthroplasty: a randomised double-blind trial. Br J Anaesth. 2022;128(1):150-158. doi:10.1016/j.bja.2021.10.001

6. Lee S, Rooban N, Vaghadia H, Sawka AN, Tang R. A randomized non-inferioritytrial of adductor canal block for analgesia after total knee arthroplasty: single injection versus catheter technique. J Arthroplasty. 2018;33(4):1045-1051. doi:10.1016/j.arth.2017.11.018

7. Mancel L, Van Loon K, Lopez AM. Role of regional anesthesia in Enhanced Recovery After Surgery (ERAS) protocols. Curr Opin Anaesthesiol. 2021;34:616-625. doi:10.1097/ACO.0000000000001048

8. Stark PA, Myles PS, Burke JA. Development and psychometric evaluation of a postoperative quality of recovery score: the QoR-15. Anesthesiology. 2013;118:1332-1340. doi:10.1097/ALN.0b013e318289b84b

Dove Medical Press encourages responsible, free and frank academic debate. The content of the Drug Design, Development and Therapy 'letters to the editor' section does not necessarily represent the views of Dove Medical Press, its officers, agents, employees, related entities or the Drug Design, Development and Therapy editors. While all reasonable steps have been taken to confirm the content of each letter, Dove Medical Press accepts no liability in respect of the content of any letter, nor is it responsible for the content and accuracy of any letter to the editor.

Drug Design, Development and Therapy

Dovepress

\section{Publish your work in this journal}

Drug Design, Development and Therapy is an international, peer-reviewed open-access journal that spans the spectrum of drug design and development through to clinical applications. Clinical outcomes, patient safety, and programs for the development and effective, safe, and sustained use of medicines are a feature of the journal, which has also been accepted for indexing on PubMed Central. The manuscript management system is completely online and includes a very quick and fair peer-review system, which is all easy to use. Visit http://www.dovepress.com/testimonials.php to read real quotes from published authors.

Submit your manuscript here: https://www.dovepress.com/drug-design-development-and-therapy-journal 